\title{
Reliability and Validity of a Modified MEDFICTS Dietary Fat Screener in South African Schoolchildren Are Determined by Use and Outcome Measures
}

Friedeburg Anna Maria Wenhold, PhD; Una Elizabeth Maclntyre, PhD; Paul Rheeder, PhD

\author{
Keywords: \\ Reliability \\ Validity \\ Diet \\ Children \\ Assessment
}

\begin{abstract}
Background In South Africa, noncommunicable diseases and obesity are increasing and also affect children. No validated assessment tools for fat intake are available. Objective To determine test-retest reliability and relative validity of a pictorial modified meats, eggs, dairy, fried foods, fats in baked goods, convenience foods, table fats, and snacks (MEDFICTS) dietary fat screener.
\end{abstract}

Design We determined test-retest reliability and diagnostic accuracy with the modified MEDFICTS as the index test and a 3-day weighed food record and parental completion of the screener as primary and secondary reference methods, respectively. Participants/setting Grade-six learners (aged 12 years, 4 months) in an urban, middleclass school $(n=93)$ and their parents $(n=72)$.

Outcome measures Portion size, frequency of intake, final score, and classification of fat intake of the modified MEDFICTS, and percent energy from fat, saturated fatty acids, and cholesterol of the food record.

Statistical analyses For categorical data agreement was based on kappa statistics, McNemar's test for symmetry, and diagnostic performance parameters. Continuous data were analyzed with correlations, mean differences, the Bland-Altman method, and receiver operating characteristics.

Results The classification of fat intake by the modified MEDFICTS was test-retest reliable. Final scores of the group did not differ between administrations $(P=0.86)$. The correlation of final scores between administrations was significant for girls only $(r=0.58 ; P=0.01)$. Reliability of portion size and frequency of intake scores depended on the food category. For girls the screener final score was significantly $(P<0.5)$ correlated to total, saturated fat, and cholesterol intakes (but not to percentenergy from fat and saturated fatty acids intakes). The sensitivity of the modified MEDFICTS was very high (>90\%), but chance corrected agreement between the classifications was poor. Parents did not agree with their children. Conclusions Test-retest reliability and relative validity of a modified MEDFICTS dietary fat screener in South African schoolchildren depended on the use and outcome measures applied.

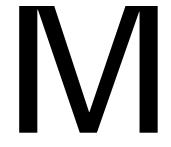

ORBIDITY AND MORTALITY FROM NONCommunicable diseases (NCDs) in South Africa are rising at a concerning rate and a large proportion of the national burden of disease is attributable to high total blood cholesterol levels. ${ }^{2}$ overweight and obesity affect a large portion of the adult population ${ }^{3}$ and are also emerging among children, particularly white children. ${ }^{4,5}$ Prevention is critical because childhood obesity may continue into adulthood. ${ }^{6}$ High fat intake is a risk

STATEMENT OF POTENTIAL CONFLICT OF INTEREST: No potential conflict of interest was reported by the authors.

FUNDING/SUPPORT: There is no funding to disclose.

ACKNOWLEDGEMENTS : The authors thank J. Stokes-Waller and $M$. Vermaak for practical development of the screener and $M$. van der Linde and J. Grimbeeck for data analysis and statistical advice factor for NCDs and for the development of obesity. Schools are well suited for nutrition programs, ${ }^{7}$ yet the South African National School Nutrition Programme currently focuses on food insecurity by targeting disadvantaged schools through the provision of a meal, promotion of sustainable food production, and nutrition education. ${ }^{8}$ The prevention of childhood obesity and NCDs is largely initiated through other interventions. ${ }^{9}$

The National Cholesterol Education Program is a program of the National Heart, Lung, and Blood Institute of the US Department of Health and Human Services. ${ }^{10}$ The National Cholesterol Education Program guidelines are part of dietetics training throughout South Africa. The meats, eggs, dairy, fried foods, fats in baked goods, convenience foods, table fats, and snacks (MEDFICTS) dietary fat screener, being freely 
available and included in many textbooks, offers a base for assessing adherence to the fat intake guidelines and for targeted, food-based dietary guidance. Numerous validation studies of the MEDFICTS screener ${ }^{11-15}$ add to its credibility, yet none has been conducted in South Africa or included children. This prompted the development of a modified MEDFICTS intended for South African primary schoolchildren.

Reliability and validity testing of screeners is usually limited to judgments based on the performance of their final classification as high or low risk. This restricts evidencebased use and refinement of the screeners, because little is known about their error structure. Because screening results are also intended as client-relevant starting points for foodbased nutrition promotion, not only the final classification should be consistent and true, but also the results pertaining to frequency of intake and portion size estimations across the foods measured.

The aim of our study was to determine the test-retest reliability and relative validity of a modified MEDFICTS dietary fat screening tool in grade-six learners in a South African school. Test-retest reliability was defined as the reproducibility of various outcome measures of the modified MEDFICTS tool when administered twice to the same participants. Relative validity was seen as the extent to which fat intake as reflected by the modified MEDFICTS dietary fat screener (the index test) agreed with percentages of energy from fat (PFE), saturated fatty acids (PSFE), and/or cholesterol intake as determined by a 3-day weighed record (primary reference method outcome measures), and the simultaneous agreement with parental assessment of the child's fat intake using the modified MEDFICTS tool (secondary reference method). Diagnostic accuracy is a related term, referring to the amount of agreement between information from the index test and the reference method(s). ${ }^{16}$

\section{STUDY DESIGN AND METHODS}

\section{Study Design}

A test-retest reliability and diagnostic accuracy study ${ }^{16}$ with a modified MEDFICTS dietary fat screener as the index test and two reference methods was conducted. A 3-day weighed food record represented a dietary assessment with an inherently different error structure than the index test with its food frequency questionnaire format, whereas parental completion of the screener was an external data source for convergent validity. ${ }^{17}$

\section{Study Population and Ethics}

All 108 children of three grade-six classes of a middle-class, predominantly white, Afrikaans-speaking public primary school in urban Pretoria were eligible. We aimed for a sensitivity and specificity of at least $80 \%$ and $60 \%$, respectively. With 27 positive and 27 negative responses, significant results would be obtained at a 95\% confidence level and a power of $80 \%$. For the test-retest reliability, a random subgroup was identified from each class.

The relevant authorities approved the study. Informed parental consent and child assent were obtained. A (unannounced) pen and a snack were given to the children after data collection. The Research Ethics Committee of the Faculty of Health Sciences (University of Pretoria) approved the study.
Modified MEDFICTS (Index Test): Development and Administration

Local registered dietitians developed the modified MEDFICTS dietary fat screener as a pictorial, South Africanized version, adapted for schoolchildren (Table 1 ). The low-fat meats category such as low-fat luncheon meat was excluded because of limited local availability and presumed lack of discriminative food knowledge in children. Photographs of example foods items were combined in one composite image per food category. Portion size estimation aids were added (Table 1). For intake frequency a "per day" option was included. Modifications to the MEDFICTS dietary fat screener were pilot tested in four separate developmental evaluation studies in a comparable school.

Participants were divided into nine groups of about 12 children each. Standardized data collection was done in school time by one researcher in the school conference room with a U-shaped seating arrangement. For privacy, adjacent desks were separated by dividers. A coded answer sheet, a colored cover sheet, and a pen were on each desk. A poster version of these sheets was placed on the front wall to assist the process. Pictures of the food categories were electronically projected one after the other in an interviewer-paced approach aimed at clarity and minimizing missing responses. Preset text was read in the mother tongue of the children from the back of a hard copy flip file, which also had the corresponding food pictures. For each food category usual portion size (small, medium, or large relative to the reference) and intake frequency were asked. The children were requested to report typical ways of eating since the beginning of the year ("since you were in grade 6") reflecting a reference period of about 9 months, which was assumed to be a meaningful period for them. No value-laden comments of food likes and dislikes were allowed, yet clarifying questions were encouraged in a friendly atmosphere. Interviewing technique has been described before. ${ }^{18}$ The second administration of the modified MEDFICTS was about 6 weeks later. Total contact time per administration per group was 40 to 45 minutes (one school period).

\section{Reference Methods}

The use of 3 consecutive days of weighed food recording by grade-six learners as part of a mathematics assignment was tested and standardized during the previous year in grade-six learners taught by the same teacher who was involved in this study. One to 2 weeks after the first administration of the modified MEDFICTS, three food-recording groups were established (Thursday, Friday, and Saturday [ $\mathrm{n}=22$ ]; Tuesday, Wednesday, and Thursday [ $\mathrm{n}=49$ ]; and Sunday, Monday, and Tuesday [ $\mathrm{n}=22]$ ). Due to financial constraints a total of only 64 children weighed their food with electronic food scales provided by the researchers. These children were randomly chosen within each food-recording group. The rest of the children used home scales or received a set of household measures. Detailed, standardized training was given to each class. This included quantification, recording, and describing foods using adapted flow diagrams. ${ }^{19}$ The food record forms were color-coded and had example entries and a plastic sleeve for supporting evidence such as wrappers.

For the secondary reference method a text version of the modified MEDFICTS dietary fat screener to be completed by the parents of the grade-six children was sent to them with the informed consent documentation. 
Table 1. Categories, example foods, and portion size references in the modified MEDFICTS ${ }^{a}$ dietary fat screener

Modified MEDFICTS

category/subcategories

Meats

Eggs

Dairy, milk, whole or fat reduced

Cheese, full cream

Dessert, full cream

Fried foods

In baked goods

Convenience foods

Table fats, high fat

Snacks, high fat
Example foods pictured on composite photograph

Beef (steak, boerewors, biltong, c minced meat); pork; chicken; mutton (chops, roast); processed meat (cold meats, bacon, polony, spreads); organ meat (kidney, chicken liver) Eggs

Fresh or long-life milk (full cream or 2\%); milk powder (full cream or blends); coffee/tea creamers; condensed or evaporated milk; full cream yogurt

Hard cheese (full cream); processed; spread or wedges (full cream); cottage/cream cheese (full cream)

Milkshakes; full cream ice cream; full cream custard; full cream dessert (blancmange type)

Chips (french fries); fried vegetables (onion rings); fried chicken (whole or pieces); fried fish (hake) or fried seafood (calamari); fried meat (sausage); fried eggs

Cakes; cookies/biscuits; sweet tarts/pastries (chelsea buns, doughnuts, eclairs); savories (samoosas, ${ }^{9}$ croissants, vetkoek $\left.{ }^{\mathrm{h}}\right)$; rusks

Tins/cans (spaghetti); packaged (pasta sauces, noodles); frozen meals (pizza)

Butter; margarine, brick wrapped in paper; mayonnaise/ salad dressing; peanut butter

Chocolates; crisps and cheese puffs; regular savory crackers; peanuts
Medium portion and/or portion size estimation aids

$90 \mathrm{~mm}$ diameter circle $\left(\mathrm{D}^{\mathrm{d}}\right)$

2 eggs $\left(P^{e}\right)$

$250 \mathrm{~mL}$ cup fresh milk $\left(\mathrm{H}^{\mathrm{f}}\right)$

3 slices each $30 \times 90 \mathrm{~mm}(\mathrm{D})$; 2 wedges (D); $125 \mathrm{~mL}(\mathrm{H})$

$125 \mathrm{~mL}$ cup $(\mathrm{H})$

$90 \mathrm{~mm}$ diameter circle (D); $125 \mathrm{~mL}$ cup $(\mathrm{H})$

$120 \mathrm{~mm}$ diameter circle (D)

$100 \times 100 \mathrm{~mm}$ square (D); $250 \mathrm{~mL}$ cup $(\mathrm{H})$

$5 \mathrm{~mL}$ teaspoon $(\mathrm{H})$

1 small packet crisps (P); $50 \mathrm{~g}$ chocolate (P); $22 \mathrm{~g}$ peanuts $(P) ; 6$ crackers, high fat $(P)$

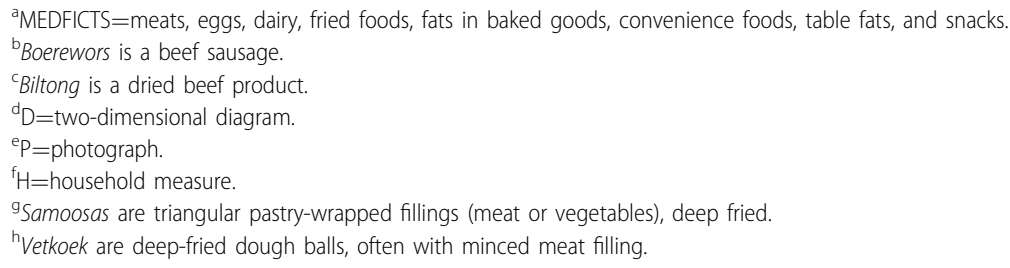

Body weight (accuracy $100 \mathrm{~g}$, Tanita electronic scale) and height (to nearest $0.1 \mathrm{~cm}$ using a portable height gauge) were obtained as part of mathematics activities by a trained teacher in a private corner of the classroom using standard techniques. Children wore single-layer summer school uniforms without footwear and jerseys.

\section{Data Analysis}

We used modified MEDFICTS scoring. Daily consumption was converted to weekly intake. This was grouped and scored as specified in the original tool ${ }^{11}$ and called the frequency of intake score. Category scores were calculated by multiplying the portion size score $(1,2$, or 3 for small, medium, and large respectively, as in the original MEDFICTS ${ }^{11}$ ) with the frequency of intake score. The sum of the category scores was the final score. If the final score (maximum=210) exceeded 68 , the modified MEDFICTS classification was "high fat."
Conversely it was "prudent." This same scoring applied to the parental completion of the screener.

For the test-retest reliability sampling bias was checked with Wilcoxon's rank sum test. Agreement between the two administrations of portion size and frequency of intake scores was expressed as percentage of pairs with perfect agreement, kappa (interpreted according to Altman ${ }^{20}$ ), and McNemar's test for symmetry. Spearman correlation coefficients were computed between the final scores. Wilcoxon's signed rank test assessed the significance of the difference between the first and the second administration final scores, supplemented by the Bland-Altman method. ${ }^{20}$

The 3-day food records were analyzed by an experienced registered dietitian using the FoodFinder3 food composition database of the South African Medical Research Council and food wrapper information. She was of the same culture as the children and familiar with Afrikaans children's eating habits, 
language use, and trends in the food industry. Food records were classified as "high fat" when mean daily PFE $>30$, PSFE $>10$, or cholesterol intake $\geq 300 \mathrm{mg}$. Conversely, diets were called "prudent." A variable "ANY" indicated that any one of the outcome measures (either PFE, PSFE, or cholesterol intake) applied. "ALL" meant that all three outcome measures had to be met simultaneously.

Mean daily energy intake was checked against presumed energy requirements by calculating basal metabolic rate using the World Health Organization formulae for persons aged 10 to 18 years. ${ }^{21}$ Physical activity level (PAL) was the ratio of mean daily energy intake to basal metabolic rate, ${ }^{22}$ calculated for each child individually. Recording fatigue was checked by comparing mean energy intake of each of the three days of recording. For weekend-day effect the two food-recording groups with a weekend day were combined and Friedman two-way analysis of variance with multiple comparisons was performed. The mean overall PAL $(\mathrm{n}=93)$ was $1.45 \pm 0.4$ (boys and girls $1.49 \pm 0.4$ and $1.41 \pm 0.4$, respectively). Twelve children had a PAL below 1.06, a value used to test whether reported energy intake from a 3-day record is plausible. ${ }^{23}$ Overall there was a significant difference in total energy intake between the days $(P=0.03)$ when comparing Day 1 with Day 3. Within the Thursday to Saturday and the Sunday to Tuesday food-recording groups there was no significant difference in energy intake across the 3 days, but in the Tuesday to Thursday group the difference was statistically significant $(P=0.04)$ in that Day 1 differed from Day 3.

For the relative validation, the final score of the first administration of the modified MEDFICTS dietary fat screener was related to the continuous measures of fat intake from the food records using Spearman rank correlation. On the basis of the modified MEDFICTS classification on the one hand and the classified outcome measures of fat intake from the food records on the other, percent classification agreement, chance corrected agreement (kappa), and parameters of diagnostic performance were determined. ${ }^{16,17}$ Agreement among the classifications by the modified MEDFICTS dietary fat screener, the food record outcome measures, and the parental screening was performed graphically with Venn diagrams.

Receiver operating characteristics (ROC) curves were created by calculating, for each outcome measure of fat intake, the sensitivity and specificity of every observed final score and plotting the sensitivity against $1-$ specificity. The area under the ROC curve reflected the discriminatory performance of the modified MEDFICTS dietary fat screener relative to the various outcome measures of fat intake.

All data were analyzed with SAS software (mainframe version 8.2, 2001, SAS Institute Inc), except the kappa, certain McNemar, and the Friedman statistics, which were done using BMDP statistical software (release 7.1, 1993, University California Press). For body mass index (BMI) for age, the Centers for Disease Control 2000 growth references were used. ${ }^{24}$ Age (in months) was calculated from date of assessment and date of birth from school records.

\section{RESULTS}

\section{Participants}

In total of 101 out of 108 children completed the modified MEDFICTS without missing values. The sample for the test-retest reliability analysis included 39 children (three groups of 13 each). Nonconsent or absenteeism resulted in loss of four children. Seven food records were discarded because of illness, suspect, and/or incomplete (at least one full day) recording. A total of 93 children had a complete modified MEDFICTS and usable food records for the primary validation. Seventy-eight parents returned a modified MEDFICTS. Six parental responses were excluded because of missing values, resulting in a sample of 72 for the final, triangular comparison. Table 2 summarizes the study population, showing that the mean BMI-for-age $z$ score was positive. Mean age was about 12 years, 4 months.

\section{Test-Retest Reliability}

Within the first administration of the modified MEDFICTS dietary fat screener there was no significant difference between children who finished both administrations and those who completed the first only $(P>0.05$ for all category and final scores; data not shown), ruling out sampling bias. Table 3 shows the agreement in both administrations of portion size and frequency of intake scores. Following adjustment for chance agreement, table fats, convenience

Table 2. Characteristics of the population studied to determine test-retest reliability and relative validity of a pictorial modified MEDFICTS ${ }^{a}$ dietary fat screener, by assessment

\begin{tabular}{lc} 
& Characteristic \\
\hline Age & BMI $^{\mathrm{b}}$-for-age \\
(mo) & $z$ score \\
\hline
\end{tabular}

mean \pm standard deviation

Reliability assessment (Test-retest)
Boys $(n=19)$
$148.9 \pm 4.4$
$0.64 \pm 1.3$
Girls $(n=20)$
$147.8 \pm 3.5$
$0.25 \pm 1.0$
Total $(n=39)$
$148.1 \pm 3.9$
$0.38 \pm 1.2$

Primary validity assessment (Modified MEDFICTS vs food records)
Boys $(n=43)$
$149.0 \pm 4.6$
$0.40 \pm 1.2$
Girls ( $n=50)$
$147.5 \pm 3.9$
$0.43 \pm 1.1$
Total $(n=93)$
$148.2 \pm 4.3 \quad 0.42 \pm 1.1$

\section{Venn diagram assessment} (Modified MEDFICTS vs food records vs parental completion of screener)

$\begin{array}{lll}\text { Boys }(n=34) & 148.0 \pm 4.7 & 0.26 \pm 1.1 \\ \text { Girls }(n=38) & 149.3 \pm 4.1 & 0.73 \pm 1.2 \\ \text { Total }(n=72) & 148.6 \pm 4.4 & 0.51 \pm 1.2\end{array}$

${ }^{a}$ MEDFICTS=meats, eggs, dairy, fried foods, fats in baked goods, convenience foods, table fats, and snacks.

${ }^{\mathrm{b}} \mathrm{BMl}=$ body mass index. 
Table 3. Test-retest reliability of portion size and frequency of intake scores of modified MEDFICTS ${ }^{\mathrm{a}}$ dietary fat screener categories $(n=39)$

\begin{tabular}{|c|c|c|c|c|c|c|c|c|}
\hline \multirow[b]{3}{*}{ Modified MEDFICTS category } & \multicolumn{4}{|c|}{ Portion Size } & \multicolumn{4}{|c|}{ Frequency of Intake } \\
\hline & \multirow{2}{*}{$\begin{array}{l}\text { Identical } \\
(\%)\end{array}$} & \multicolumn{2}{|c|}{$\kappa$} & \multirow{2}{*}{$\begin{array}{l}\text { McNemar } \\
P \text { value }\end{array}$} & \multirow{2}{*}{$\begin{array}{l}\text { Identical } \\
(\%)\end{array}$} & \multicolumn{2}{|c|}{$\kappa$} & \multirow{2}{*}{$\begin{array}{l}\text { McNemar } \\
P \text { value } \\
\end{array}$} \\
\hline & & Value & $P$ value & & & Value & $P$ value & \\
\hline Meat & 65.8 & $0.36^{\mathrm{b}}$ & 0.01 & 0.04 & 82.1 & $0.53^{c}$ & 0.0001 & 0.06 \\
\hline Eggs & 70.6 & $0.43^{c}$ & 0.01 & 0.37 & 82.1 & $0.55^{\mathrm{c}}$ & 0.0001 & 0.51 \\
\hline Dairy, milk, high fat & 43.2 & $0.10^{d}$ & 0.40 & 0.44 & 87.2 & $0.12^{d}$ & 0.30 & 0.51 \\
\hline Dairy, cheese, high fat & 65.7 & $-0.15^{d}$ & 0.26 & 0.84 & 48.7 & $0.14^{d}$ & 0.21 & 0.06 \\
\hline Dairy, dessert, high fat & 70.3 & $0.28^{\mathrm{b}}$ & 0.16 & 0.37 & 56.4 & $0.19^{d}$ & 0.11 & 0.35 \\
\hline Fried foods & 76.3 & $0.19^{d}$ & 0.19 & 0.10 & 48.7 & $0.08^{d}$ & 0.53 & 0.72 \\
\hline In baked goods & 71.8 & $0.34^{\mathrm{b}}$ & 0.004 & 0.15 & 61.5 & $0.36^{\mathrm{b}}$ & 0.002 & 0.04 \\
\hline Convenience foods & 72.2 & $0.48^{c}$ & 0.001 & 0.26 & 61.6 & $0.30^{\mathrm{b}}$ & 0.02 & 0.51 \\
\hline Table fats, high fat & 75.0 & $0.52^{c}$ & 0.002 & 0.32 & 84.6 & $0.17^{d}$ & 0.27 & 0.41 \\
\hline Snacks, high fat & 48.7 & $0.08^{d}$ & 0.51 & 0.62 & 76.9 & $0.46^{c}$ & 0.002 & 0.34 \\
\hline
\end{tabular}

${ }^{a}$ MEDFICTS=meats, eggs, dairy, fried foods, fats in baked goods, convenience foods, table fats, and snacks

${ }^{b}$ Fair agreement $(\kappa=0.21-0.40)$.

${ }^{c}$ Moderate agreement $(\kappa=0.41-0.60)$.

${ }^{\mathrm{d}}$ Poor agreement $(\kappa \leq 0.20)$.

foods, and eggs had moderate portion size agreement across the two assessments. For the remaining food categories kappa was $<4$, denoting fair or poor agreement. ${ }^{20}$ Only for meat were the nonagreeing responses in the two administrations nonsymmetrical (McNemar $P<0.05$ ). This suggests that in general, when the first and the second administration did not agree, a similar proportion of children changed from smaller to larger portion size and vice versa. For frequency of intake the chance-corrected agreement was moderate for eggs, meat, and snacks, yet fair or poor for the remaining food categories. In general, for weekly consumption, those who reported higher intake in the second administration were balanced by those who reported lower intake (see McNemar information in Table 3). In summary, portion size and frequency of intake test-retest reliability differed across food categories. Where there was nonagreement in the two administrations, there tended to be random, as opposed to systematic, error.

The mean final scores in the two administrations did not differ significantly. The mean whole group difference was $0.69 \pm 32.6$ (95\% CI -9.9 to 11.3 ). There was a small, but statistically significant, correlation $(r=0.36 ; P=0.02)$ between the final scores of the two administrations. This correlation was nonsignificant $(r=0.26 ; P=0.29)$ for boys, yet highly significant for girls $(r=0.58 ; P=0.01)$. A wide scatter around the lines of equality in the Bland-Altman plots (data not shown) was noted for the group as a whole. Girls tended to be closer to these lines, suggesting better reproducibility. Based on the fat intake classification, there was agreement for 36 out of 39 (more than $90 \%$ ) of children during the two administrations. Three children were reclassified from "high fat" to "prudent" intake in the retest. It follows that statistical

Table 4. Correlation coefficients $\left(r\right.$ ) between modified MEDFICTS ${ }^{a}$ dietary fat screener final score and food record outcome measures of fat intake

\begin{tabular}{|c|c|c|c|c|c|c|}
\hline \multirow[b]{2}{*}{ Food record outcome measures of fat intake } & \multicolumn{2}{|c|}{ Boys $(n=43)$} & \multicolumn{2}{|c|}{ Girls $(n=50)$} & \multicolumn{2}{|c|}{ Total $(n=93)$} \\
\hline & $r$ & $P$ value & $r$ & $P$ value & $r$ & $P$ value \\
\hline Total fat energy & 0.03 & 0.87 & 0.30 & 0.04 & 0.16 & 0.13 \\
\hline $\mathrm{PFE}^{\mathrm{b}}$ & -0.17 & 0.28 & 0.14 & 0.35 & -0.02 & 0.82 \\
\hline Saturated fat energy & 0.06 & 0.72 & 0.31 & 0.03 & 0.20 & 0.06 \\
\hline PSFE $^{c}$ & -0.01 & 0.93 & 0.19 & 0.18 & 0.08 & 0.45 \\
\hline Cholesterol & -0.09 & 0.56 & 0.31 & 0.03 & 0.18 & 0.10 \\
\hline
\end{tabular}

${ }^{\mathrm{a} M E D F I C T S}=$ meats, eggs, dairy, fried foods, fats in baked goods, convenience foods, table fats, and snacks.

${ }^{\mathrm{b}} \mathrm{PFE}=$ percentage fat energy.

${ }^{\mathrm{C}} \mathrm{PSFE}=$ percentage saturated fatty acid energy. 
technique, sex, and the outcome measure used played a role in the test-retest reliability of the modified MEDFICTS dietary fat screener.

\section{Relative Validity}

The correlations between the modified MEDFICTS final score and fat intake from the food records are presented in Table 4. For girls the final score of the modified MEDFICTS was significantly $(P<0.05)$ related to total fat energy, total saturated fat energy, and cholesterol intake. The modified MEDFICTS classification indicated that $86(92 \%)$ of children had a high fat intake. High fat intake according to the food records ranged from 16 (17\%) when all three intake cutoffs applied simultaneously, to $84(90 \%)$ if only one of the intake cutoffs had been fulfilled. When food record PFE, PSFE, or "ANY" acted as outcome measures, the percent of true "high fat" intakes plus true "prudent" intakes (ie, perfect agreement between the modified MEDFICTS and the food record classification) was high: $72 \%, 83 \%$, and $85 \%$, respectively. This was in contrast to cholesterol intake or meeting all three food record outcome measures of fat intake, where many false classifications into "high fat" intake by the modified MEDFICTS dietary fat screener were found.

Parameters of diagnostic accuracy of the modified MEDFICTS dietary fat screener relative to fat intake from the food records are shown in Table 5. The sensitivity always exceeded 0.9 , yet the specificity was always low, with performance relative to PSFE being highest $(0.20)$. The overall and the positive predictive values exceeded 0.7 for PFE, PSFE, and when any of the three food record outcomes met the cutoff, but for cholesterol and when all three outcome measures had to be met they were low. This was also true for the prevalence rates. Conversely, when cholesterol intake was part of an outcome measure the negative predictive value was highest. The odds ratio for PSFE was much higher than any one of the other two outcome measures or combinations, yet the likelihood ratios below 2 suggest minimal increase in the likelihood of high fat intake. ${ }^{17}$ The high sensitivity of the modified MEDFICTS dietary fat screener suggested that it was able to correctly identify those children with intakes categorized as "high fat," but this was at the expense of its ability to correctly identify those with fat intakes categorized as "prudent." The presence of cholesterol as an outcome measure of high fat intake in the food record played a role.

When the modified MEDFICTS dietary fat screener was completed by parents, the percentage identical classifications into "high fat" or "prudent" categories was 72\% (52 out of 72). When corrected for chance the agreement was poor $(\kappa=0.16)$. The McNemar test indicated a departure from symmetry $(P=0.0010)$; parents had consistently lower scores than their children.

The modified MEDFICTS classification completed by the children was simultaneously compared with their food record classifications and the parental completion of the screener through the use of Venn diagrams. The Figure illustrates the classification agreement among the three methods. The Figure also shows that concurrent classification of "prudent" intake (nonshaded, dashed circle) was rare. When either PFE (Figure, panel A), or PSFE (Figure, panel B), 


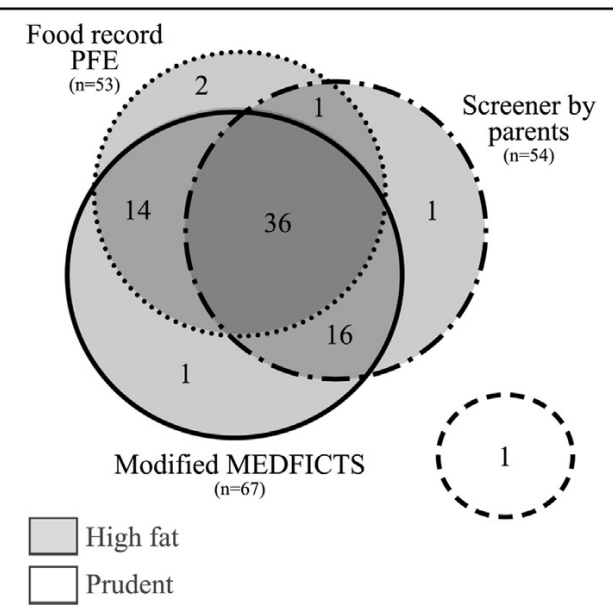

A Modified MEDFICTS ${ }^{\mathrm{a}}$, food record $\mathrm{PFE}^{\mathrm{b}}$ and screener by parents

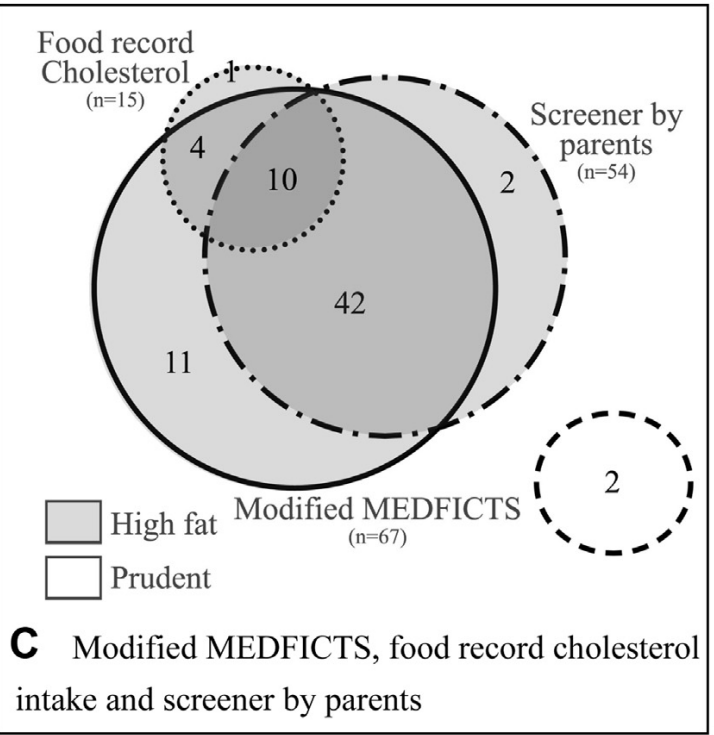

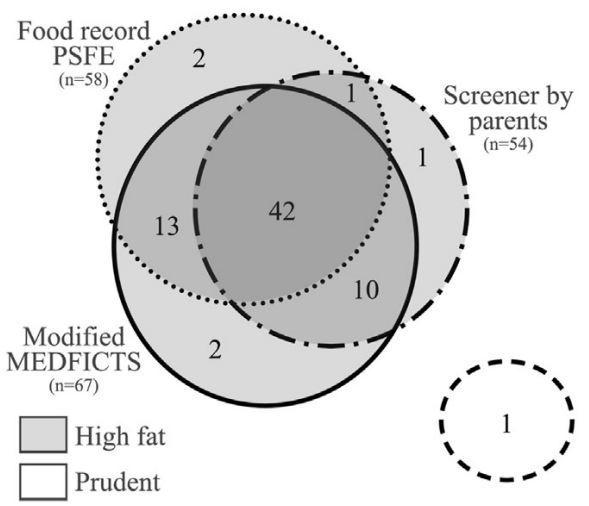

B Modified MEDFICTS, food record PSFE ${ }^{\mathrm{C}}$ and screener by parents

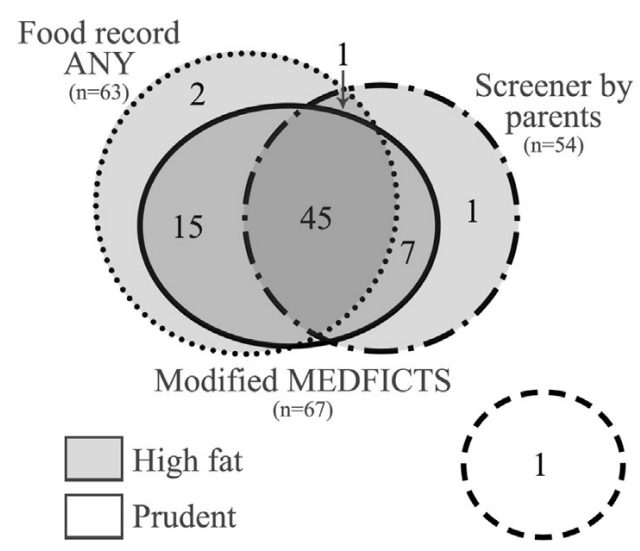

D Modified MEDFICTS, any fat intake outcome from food record, and screener by parents

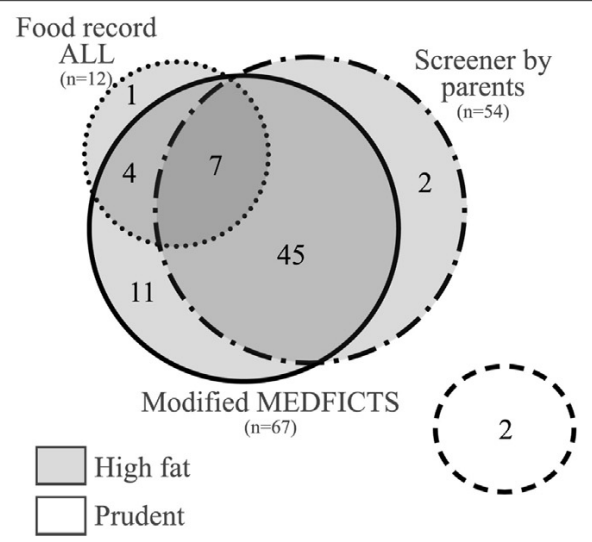

E Modified MEDFICTS, all outcomes from food record simultaneously, and screener by parents

Figure. Triangular classification agreement: Modified MEDFICTS dietary fat screener (solid-line circle), food record (dotted-line circle), and screener by parents (dash-dot-line circle) $(n=72)$. ${ }^{a}$ MEDFICTS=meats, eggs, dairy, fried foods, fats in baked goods, convenience foods, table fats, and snacks. ${ }^{b} \mathrm{PFE}=$ percentage fat energy. ${ }^{\mathrm{C}} \mathrm{PSFE}=$ percentage saturated fatty acid energy. 
or cholesterol (Figure, panel C) acted as outcome measure, then the perfect agreement among all three methods in terms of "high fat" intake ranged from 10 (14\%) for cholesterol to 42 (59\%) for PSFE (darkest shading; ie, triple overlaps in the Venn diagrams). For 52 cases (73\%) the modified MEDFICTS and the parental completion of the screener resulted in an identical "high fat" classification. The agreement regarding high fat intake between the modified MEDFICTS and the food record classification ranged from $14(20 \%)$ for cholesterol intake as outcome measure (Figure, panel C), to $60(85 \%)$ when any of the three outcome measures (Figure, panel D) could apply.

The areas under the ROC curve ranged from 0.545 for PFE, 0.548 for cholesterol, 0.555 for "ANY," 0.604 for "ALL," to 0.654 for PSFE as outcome measure. The sensitivity (true positive rate) and the false positive rate (1 - specificity) covaried when the cutoff point of the modified MEDFICTS dietary fat screener was changed. Over the five curves, the sum of sensitivity and specificity was highest at modified MEDFICTS final score cutoffs of 98 and 118. The effect of changing the MEDFICTS cutoff value to 98 and 118 on the sensitivity and the specificity relative to each outcome of fat intake was tested (data not shown). The sum of the sensitivity and specificity was similar at the two cutoffs; cutoff 98 resulted in higher sensitivity and 118 favored specificity.

\section{DISCUSSION}

The importance of reliable and valid measurement of dietary intake is widely acknowledged, yet dietary assessment remains challenging. Methodologic and context factors play a role. We examined the test-retest reliability and the validity of a South Africanized, pictorial, child-appropriate MEDFICTS dietary fat screener relative to a 3-day weighed food record as a school mathematics assignment and parental completion of the MEDFICTS screener.

Dietary screeners have different uses. If used to classify individuals into those with and without high fat intake, then test-retest reliability of the classification is important. The very high percentage of identical classifications in the two administrations suggests consistent discriminative properties of the modified MEDFICTS dietary fat screener (eg, to identify those who need intervention).

If, however, the screener is used for comparing, ranking, or monitoring individuals or groups, then reproducibility of the final score may be preferable for describing test-retest reliability. The final score of the modified MEDFICTS exhibited moderate test-retest reliability $(r=0.36 ; P=0.02)$ in the grade-six children as a group. This correlation is similar to some ${ }^{25}$ yet lower than other, ${ }^{26}$ test-retest studies in children, but considerable differences in research contexts, study designs, and outcome measures can explain this. Previous review studies have shown that reliability correlations for food frequency questionnaires among school-age children vary considerably, ${ }^{27,28}$ confirming instrument and setting effects. Our finding that the reliability coefficient was nonsignificant for boys yet highly significant for girls suggested a nonequal distribution of the measurement error among participants. It is possible that girls are more exposed to and aware of food than boys, resulting in more consistent reporting.

Differences across food categories, portion size, and frequency of intake estimates can explain final score reliability and these are important when the screener is used to direct nutrition education. Chance-corrected agreement was poor for almost half of the food categories, yet overall, higher intakes in the second administration were balanced by reports of lower intakes. The kappas (Table 3) are substantially lower than those reported by Smith and colleague ${ }^{29}$ where children completed a food checklist twice on the same day. Jonsson and colleagues $^{30}$ considered a $58 \%$ to $85 \%$ agreement between four breakfast food choices 8 weeks apart as "good." These authors explain the cases of unreliability in terms of a combination of random and systematic error. As evident from the discussion set out below, both types of error may also apply to our study.

Sometimes methods are deemed reliable because subsequent administrations are not significantly different. ${ }^{26,31}$ In most studies among schoolchildren the first measurement of dietary intake gave higher values than the second. ${ }^{27}$ Here our findings tended to differ. Whereas the small mean difference in final score indicates that the two administrations of the modified MEDFICTS agreed well on average, the variability suggests that for an individual absolute agreement was less likely. This was confirmed by the Bland-Altman method. For girls there tended to be less variability (less random error), but a bias (systematic error) toward lower scores in the second administration.

Because test-retest reliability is a prerequisite of but no guarantee for validity, the reliability results may affect the validity analysis. In the absence of a gold standard, the appropriateness and credibility of the reference method should be established. Because the error structure of food records and food frequency questionnaires differ, ${ }^{32}$ we considered multiday food records an appropriate reference. The food recording was integrated into mathematics assignments, in this way creating a win-win situation for research and teaching. The mean PAL suggested that, on average, the energy intake was plausible for the 3-day recording period, yet it could not be regarded as representing long-term habitual diets. ${ }^{33}$ Recording fatigue, evidenced by overall lower mean energy intakes on Day 3 compared with Day 1 , could have played a role. Our data tentatively suggest that inclusion of a weekend day in the food recording by schoolchildren possibly counteracted recording fatigue, but this requires further investigation. Finally, the BMI-for-age $z$ scores suggested that the children were, on average, heavier than the American reference population, one of the many variables known to be associated with underreporting. ${ }^{34-37}$

Against the backdrop of limited test-retest reliability of the modified MEDFICTS in the group as a whole and specifically among boys the lack of significant correlations between the final score in the modified MEDFICTS and the PFE, PSFE, and cholesterol in the food records was not surprising. Thus, only for girls could a meaningful relative validity be reasonably expected. The finding that total fat intakes (rather than energy contributions) revealed significant correlations has previously been documented and explained. ${ }^{38,39}$ Because the reference method is imperfect, a correlation coefficient may underestimate the level of agreement (attenuation bias) with actual usual intake. The correlation coefficients thus aid comparison to previous research. The usefulness of a combination of statistical methods in dietary validation that was demonstrated in this study has been highlighted by other researchers. ${ }^{28,40}$

The diagnostic accuracy of the modified MEDFICTS dietary fat screener points to higher sensitivity at the expense of 
specificity. For this reason the modified MEDFICTS dietary fat screener performed well in correctly identifying those children who had high fat intakes, but was not able to correctly identify those with prudent fat intakes. Mochari and colleagues ${ }^{15}$ also found higher sensitivity (0.86) and lower specificity (0.57) in the original MEDFICTS instrument, with the latter being significantly worse for women. They recommended recalibration to improve specificity, but in our study changing the cutoff for classifying final scores as "high fat" or "prudent" did not achieve the ultimate goal of acceptable sensitivity and specificity. Thus reworking the components of the modified MEDFICTS dietary fat screener could increase its validity. The process could start with the food categories. Teal and colleagues ${ }^{14}$ in their work with African-American women suggested that the addition of a mixed food category could improve the performance of the original MEDFICTS instrument. Knowledge of the sources of dietary fat of South African schoolchildren is a prerequisite for such a change, something not yet available on national level. Other refinements would involve portion size estimation, which remains a major challenge in dietary assessment ${ }^{41}$ and the measurement of frequency of intake. Also the scoring system presents refinement opportunities. The poorer performance of the modified MEDFICTS dietary fat screener when compared with food record outcome measures that included cholesterol may be related to the inability of a 3-day record to capture true cholesterol intake, which is characterized by day-to-day variation. ${ }^{42}$ We have published the details about the lack of agreement between the children and their parents ${ }^{18}$ but the concurrent agreement with outcome measures of fat intake from the weighed food record emphasizes the complexity of determining what is true. Conclusions from other review studies about the involvement of parents are inconsistent. ${ }^{28,35}$

Dietary assessment is critical yet remains challenging, particularly amongst children, where cognitive factorsspecifically to record, remember, or generalize intake-as well as restricted knowledge of food and food preparation, limited motivation, and attention span are well-documented child-specific respondent factors that can contribute to error. ${ }^{27,28,37}$ True inter- and intraindividual variability are also important. In 5- to 17-year-old children the ratio of intra- to intersubject variances in intake was about twice that observed in adults. ${ }^{43}$ Fat intake and practices that increase fat intake have been shown to vary by meal and day in schoolchildren in grades four to six..$^{31}$ Our participants were homogeneous in terms of age, education level, and culture, and index test administration was very controlled, which limits generalization but also opens opportunities for screener refinement. The very high prevalence of high fat intakes (Table 5) was unexpected, affecting the power of our study. Because predictive values change with prevalence, it is likely that the positive predictive value of the modified MEDFICTS dietary fat screener will decrease if the prevalence of high fat intake is lower. Equally the negative predictive value is expected to increase. ${ }^{17}$ Thus, the predictive values cannot be generalized. The lack of agreement with the parental assessment highlight that for assessing children's fat intake numerous specific factors must be considered: food choices like snacks and fast foods are made outside the home by the children themselves, whereas for others, such as table fats and dairy, children eat what is provided to them by their parents. Because school lunches were not served in the school we studied, this was not a source of error.

\section{CONCLUSIONS}

The modified MEDFICTS dietary fat screener builds on international experience regarding dietary fat screening methodology and at the same time takes local circumstances and the specific target group into consideration. The study contributes to describing the type and magnitude of error of the modified MEDFICTS dietary fat screener in assessing fat intake and in this way provides a basis for appropriate instrument use and refinement. Test-retest reliability was demonstrated for the modified MEDFICTS classification. The final score showed test-retest reliability for groups of children. Boys were characterized by random error and lack of reproducibility, whereas for girls test-retest reliability using the final score was established. Portion size estimation and frequency of intake posed challenges yet differed by food category. The 3-day food record appeared to be a plausible reflection of energy intake during the recording period, making it an acceptable primary reference method in the validation. For girls a significant correlation between the modified MEDFICTS final score and intake of total fat, saturated fatty acids, and cholesterol was established. Parental screening of the fat intake of their children differed from the children's selfassessment. The sensitivity of the modified MEDFICTS classification was very high, but chance-corrected agreement was poor. Changing the cutoff did not improve diagnostic performance that reflected high sensitivity and high specificity simultaneously.

Our previous work ${ }^{18}$ showed that the modified MEDFICTS was internally consistent when used by parents to describe their children, and when used by the children themselves. In our current study it emerged that the outcome measures (ie, which "score" in the modified MEDFICTS and in the food record are used in the assessment of reliability and validity) determine the result. Girls tend to perform better. We argue that different uses of a screener require different outcome measures. The findings in our study regarding test-retest reliability and relative validity therefore suggest that the intended use determines the performance of the modified MEDFICTS dietary fat screener. It should not yet be used as sole indicator of fat intake of individual South African schoolchildren; its possible value amongst South African schoolchildren currently lies in creating awareness of high fat intakes (even though some intakes may be incorrectly classified as high fat). Because the modified MEDFICTS dietary fat screener, the food record, and the parental assessment of the children suggest high fat intakes, primary prevention programs and refined measurement of fat intake are urgently needed.

\section{References}

1. Moyasi BM, Flisher A, Lalloo UG, et al. The burden of noncommunicable diseases in South Africa. Lancet. 2009;374(9693): 934-947.

2. Norman R, Bradshaw D, Steyn K, et al. Estimating the burden of disease attributable to high cholesterol in South Africa in 2000. S Afr Med J. 2007;97(8 pt 2):708-715.

3. Department of Health, Medical Research Council. South Africa Demographic and Health Survey 2003 Full Report. Pretoria, South Africa: Department of Health; 2007. 
4. Rossouw H, Grant CC, Viljoen M. Overweight and obesity in children and adolescents: The South African problem. S Afr J Sci. 2012;108(5/6), Art. \#907, 7 pages. http://dx.doi.org/10.4102/sajs. v108i5/6.907.

5. Armstrong MEG, Lambert MI, Lambert EV. Secular trends in the prevalence of stunting, overweight and obesity among South African children (1994-2004). Eur J Clin Nutr. 2011;65(7):835-840.

6. Lloyd LJ, Langley-Evans SC, McMullen S. Childhood obesity and risk of the metabolic syndrome: A systematic review. Int J Obesity. 2012;36(1):1-11.

7. Wenhold FAM, Kruger S, Muelhof E. Nutrition for school-age children and adolescents. In: Steyn NP, Temple N, eds. Community Nutrition Textbook for South Africa: A Rights-Based Approach. Tygerberg, South Africa: Food and Agriculture Organization and Medical Research Council; 2008:441-478.

8. National Guidelines for the Implementation, Monitoring and Reporting on the National School Nutrition Programme. Draft 5. Pretoria, South Africa: South African Department of Education; 2004.

9. Draper CE, de Villiers A, Lambert EV, et al. HealthKick: A nutrition and physical activity intervention for primary schools in low-income settings. BMC Public Health. 2010;10:398.

10. National Cholesterol Education Program (NCEP) Expert Panel on Detection, Evaluation, and Treatment of High Blood Cholesterol in Adults (Adult Treatment Panel III). Third Report of the National Cholesterol Education Program (NCEP) Expert Panel on Detection, Evaluation, and Treatment of High Blood Cholesterol in Adults (Adult Treatment Panel III): Final report. Circulation. 2002;106(25):3143-3421.

11. Kris-Etherton P, Eisenstat B, Jaax S, et al. Validation for MEDFICTS, a dietary assessment instrument evaluating adherence to total and saturated fat recommendations of the National Cholesterol Education Program Step 1 and Step 2 diets. J Am Diet Assoc. 2001;101(1):81-86.

12. Taylor AJ, Wong $\mathrm{H}$, Wish $\mathrm{K}$, et al. Validation of the MEDFICTS dietary questionnaire: A clinical tool to assess adherence to American Hear Association dietary fat intake guidelines. Nutr J; 2003. June 13:2-4.

13. Holmes AL, Sanderson B, Maisiak R, Brown A, Bittner V. Dietitian services are associated with improved patient outcomes and the MEDFICTS dietary assessment questionnaire is a suitable outcome measure in cardiac rehabilitation. J Am Diet Assoc. 2005;105(10):1533-1540.

14. Teal CA, Baham DL, Gor BJ, Jones LA. Is the MEDFICTS rapid dietary fat screener valid for premenopausal African-American women? J Am Diet Assoc. 2007;107(5):773-781.

15. Mochari H, Gao Q Mosca L. Validation of the MEDFICTS dietary assessment questionnaire in a diverse population. J Am Diet Assoc. 2008:108(5):817-822

16. Bossuyt PM, Reitsma JB, Bruns DE, et al. Towards complete and accurate reporting of studies of diagnostic accuracy: The STARD initiative. Clin Chem. 2003;49(1):1-6.

17. Gleason PM, Harris J, Sheean PM, Boushey CJ, Bruemmer B. Publishing nutrition research: Validity, reliability, and diagnostic test assessment in nutrition-related research. J Am Diet Assoc. 2010;110(3):409-419.

18. Wenhold F, MacIntyre U, Rheeder P. Screening for dietary fat intake of grade six children: Self-assessment vs. maternal assessment [published online ahead of print September 24, 2012]. Maternal Child Nutr. http://dx.doi.org/10.1111/j.1740-8709.2012.00444.x.

19. Wold RS, Lopez ST, Pareo-Tubbeh SL, et al. Helping elderly participants keep 3-day diet records in the New Mexico Aging Process Study. J Am Diet Assoc. 1998;98(3):326-332.

20. Altman DG. Practical Statistics for Medical Research. London, UK: Chapman \& Hall/CRC; 2001.

21. World Health Organization. Energy and Protein Requirements: Report of a Joint FAO/WHO/UNU Expert Consultation. Geneva, Switzerland: World Health Organization; 1985.

22. McCrory MA, Hajduk CL, Roberts SB. Procedures for screening out inaccurate reports of dietary intake. Public Health Nutr. 2002;5(6A): 873-882.

23. O'Connor J, Ball EJ, Steinback KS, et al. Comparison of total energy expenditure and energy intake in children aged 6-9 y. Am J Clin Nutr. 2001;74(4):643-649.
24. Centers for Disease Control and Prevention. CDC growth charts (2000). http://www.cdc.gov/growthcharts/cdc_charts.htm. Accessed January 26, 2014.

25. Yaroch AL, Resnicow K, Petty AD, Khan LK. Validity and reliability of a modified qualitative dietary fat index in low-income, overweight, African American adolescent girls. J Am Diet Assoc. 2000;100(12): 1525-1529.

26. Metcalf PA, Scragg RKR, Sharpe S, Fitzgerald EDH, Schaaf D, Watts C. Short-term repeatability of a food frequency questionnaire in New Zealand children aged 1-14y. Eur J Clin Nutr. 2003;57(11): 1498-1503.

27. McPherson RS, Hoelscher DM, Alexander M, Scanlon KS, Serdula MK. Dietary assessment methods among school-aged children: Validity and reliability. Prev Med. 2000;31(2):S11-S33.

28. Kolodziejczyk JK, Merchant G, Norman GJ. Reliability and validity of child/adolescent food frequency questionnaires that assess food and/ or food groups. J Pediatr Gastroenterol Hepatol Nutr. 2012;55:4-13.

29. Smith KW, Hoelscher DM, Lytle LA, et al. Reliability and validity of the Child and Adolescent Trial for Cardiovascular Health (CATCH) food checklist: A self-report instrument to measure fat and sodium intake by middle school students. J Am Diet Assoc. 2001;101(6):635642. 647.

30. Jonsson I, Gummeson L, Conner M, Svensson E. Assessing food choice in school children: Reliability and construct validity of a method stacking food photographs. Appetite. 1998;30(1):25-37.

31. Weber Cullen K, Lara KM, de Moor C. Children's dietary fat intake and fat practices vary by meal and day. I Am Diet Assoc. 2002;102(12):1773-1778.

32. Kipnis V, Midthune D, Freedman L, et al. Bias in dietary-report instruments and its implications for nutritional epidemiology. Public Health Nutr. 2002;5(6A):915-923.

33. Torun B, Davies PS, Livingstone MB, Paolisso M, Sackett R, Spurr GB. Energy requirements and dietary energy recommendations for children and adolescents 1 to 18 years old. Eur J Clin Nutr. 1996;50(suppl 1):37S-81S

34. Forrestal SG. Energy misreporting among children and adolescents: A literature review. Maternal Child Nutr. 2011;7(2):112-127.

35. Burrows TL, Martin RJ, Collins CE. A systematic review of the validity of dietary assessment methods in children when compared with the methods of doubly labeled water. J Am Diet Assoc. 2010;110(10): 1501-1510.

36. Murakami K, Miyake Y, Sasaki S, Tanaka K, Arakawa M. Characteristics of under- and over-reporters of energy intake among Japanese children and adolescents: The Ryukyus Child Health Study. Nutrition. 2012;28(5):532-538.

37. Magarey A, Watson J, Golley RK, et al. Assessing dietary intake in children and adolescents: Considerations and recommendations for obesity research. Int J Pediatr Obesity. 2011;6(1):2-11.

38. Van Assema P, Brug J, Ronda G, Steenhuis I. The relative validity of a short Dutch questionnaire as a means to categorize adults and adolescents to total and saturated fat intake. J Hum Nutr Diet. 2001;14(5):377-390.

39. Rohrmann S, Klein G. Validation of a short questionnaire to qualitatively assess the intake of total fat, saturated, monounsaturated, polyunsaturated fatty acids, and cholesterol. J Hum Nutr Diet. 2003;16(2):111-117.

40. MacIntyre UE, Venter CS, Vorster HH, Steyn HS. A combination of statistical methods for the analysis of the relative validation data of the quantitative food frequency questionnaire used in the THUSA study. Publ Health Nutr. 2001;4(1):45-51.

41. Foster E, Adamson AJ, Anderson AS, Barton KL, Wrieden WL. Estimation of portion size in children's dietary assessment: Lessons learnt. Eur J Clin Nutr. 2009;63(suppl 1):S45-S49.

42. Institute of Medicine, Food and Nutrition Board. Dietary Reference Intakes for Energy, Carbohydrate, Fiber, Fat, Fatty Acids, Protein, and Amino Acids (Macronutrients). Washington, DC: National Academies Press; 2002.

43. Livingstone MBE, Robson PJ. Measurement of dietary intake in children. Proc Nutr Soc. 2000;59(2):279-293. 
Author information:

F. A. M. Wenhold is a senior lecturer and U. E. Maclntyre is a professor extraordinary, Department of Human Nutrition, and P. Rheeder is an associate professor and acting head, School of Health Systems and Public Health, all at the University of Pretoria, Pretoria, South Africa

Address correspondence to: Friedeburg Anna Maria Wenhold, PhD, Department of Human Nutrition, University of Pretoria, Private bag X323, Arcadia, Pretoria, South Africa. E-mail:

friede.wenhold@up.ac.za 